\title{
Research on GIS Monitoring System in Operation Based on Rail Robot
}

\author{
Peng-Fei Jia ${ }^{1, *}$, Hong-Yu $\mathrm{Ni}^{2}$, Xing-Hui Zhang ${ }^{3}$, Su Huang ${ }^{2}$, Li-Hua $\mathrm{Li}^{1}$ and Yang Yang ${ }^{1}$ \\ ${ }^{1}$ China Electric Power Research Institute, High Voltage Research Institute, 15 Xiaoying East Road, Qinghe, Haidian District, Beijing, \\ China \\ ${ }^{2}$ Shaoxing Power Supply Branch, Zhejiang Electric Power Co., Ltd, 58 Shengli East Road, Yuecheng District, Shaoxing, China \\ ${ }^{3}$ Equipment Management Department, State Grid Corporation Limited, 86 West Chang'an Street, Xicheng District, Beijing, China
}

\begin{abstract}
GIS is prone to various defects and faults after putting into operation, and the failure rate is high. At present, the inspection methods of GIS in operation include traditional inspection mode, twodimensional code inspection mode, radio frequency identification technology mode and robot inspection mode. However, the current inspection robot has the disadvantages of weak adaptability to complex environment, insufficient inspection scope, low efficiency and low intelligence. Therefore, this paper designs a GIS monitoring system based on the rail robot. By installing all kinds of sensors on the rail robot, with the help of binocular vision and mechanical arm, the omni-directional live detection of GIS in transit can be realized.
\end{abstract}

\section{Introduction}

The internal flashover fault of GIS equipment usually occurs within one year after installation or overhaul[ ${ }^{[1]}$. According to statistical data, the failure rate of equipment operation in the first year is 0.53 times / interval, and in the second year it drops to 0.06 times / interval, and then tends to be stable. According to the operation experience, the fault rate of disconnector and pot insulator is the highest, which is $30 \%$ and $26.6 \%$ respectively; the fault rate of bus is $15 \%$; the fault rate of voltage transformer is $11.66 \%$; the fault rate of circuit breaker is $10 \%$; the failure rate of other components is $6.74 \%{ }^{[2]}$.

With the concept of smart grid put forward, domestic and foreign power inspection has developed rapidly. At present, there are several inspection methods for GIS Substation: traditional inspection mode, two-dimensional code inspection mode, radio frequency identification technology mode, inspection robot inspection mode ${ }^{[3]}$.

Traditional inspection method: the traditional inspection method mostly uses paper records or special marks to mark the defects of power equipment ${ }^{[4]}$. QR code inspection method: this method uses mobile terminal equipment in the power system inspection, realizes the intelligent mobile inspection system based on $\mathrm{QR}$ code, and completes the intelligent inspection platform by using $3 \mathrm{G}$ mobile communication technology and web service data mining technology ${ }^{[5]}$. Inspection of radio frequency identification technology: this method uses the identification function of identification terminal to identify RFID tag, and then realizes wireless communication by using the communication between mobile terminal and server ${ }^{[6]}$. Inspection mode of inspection robot: the inspection mode is to carry each sensor module on the robot with the main controller as the core to realize the inspection of power equipment.

Practice has proved that the above inspection methods have serious deficiencies. The traditional way of power inspection is the earliest and original way of inspection, which is widely used. However, it is affected by human factors, it is not easy to supervise, and the results are difficult to report in time, and it is difficult to save and manage. The two-dimensional code inspection method has the two-dimensional code tag which needs close scanning, which cannot meet the inspection at high places or places where human resources are difficult to reach; and the two-dimensional code label is easy to be damaged and difficult to maintain ${ }^{[7]}$. At present, the inspection based on RFID mainly has the following problems Question: at present, most RFID tags are active tags, that is, the power supply needs to be changed regularly, so its maintainability is poor; Basically, it only provides solid-state location map, without real-time visible map showing inspector's position combined with GIS; in addition, it also has the problems of offline inspection, information cannot be reported in time, and no parameter information of equipment to be inspected is described. Due to the serious shortage of the above three inspection methods, robot inspection has gradually become a new type of inspection in recent years Power equipment inspection mode.

\section{PROBLEMS IN ROBOT INSPECTION}

From the practical point of view of the existing robot inspection system:

\footnotetext{
* Corresponding author: jiapengfei@epri.sgcc.com.cn
} 


\subsection{The adaptability to complex site environment is not strong}

Due to the complex working environment, the existing transmission line inspection robot cannot accurately identify all kinds of devices due to the variety of devices to maintain the stability and insulation of the line, so it cannot take the corresponding line crossing device, resulting in the line inspection task cannot be completed smoothly. However, for the substation inspection robot, it cannot respond to the environmental changes timely and accurately in the process of inspection, and cannot normally move in case of placing safety fence and personnel walking. These conditions show that the existing inspection robots are not adaptable to the complex scene environment and have insufficient ability to deal with emergencies independently.

\subsection{The scope of operation is greatly restricted}

At present, most of the substation inspection robots related to GIS live detection adopt "wheel structure". Due to its limitation, the robot can only move on the ground near the GIS equipment, and can not approach most areas of the GIS equipment. As a result, it can only realize some simple functions such as temperature detection of partial GIS shell surface and partial reading of GIS instrument parameters, which greatly limits the robot to realize other kinds of live line inspection test function.

\subsection{Low inspection efficiency}

At present, the demand of live line detection after putting into operation is not fully considered in the design stage of GIS equipment. The geometric structure of GIS equipment is complex and the sensor interface is blocked. Some GIS equipment areas cannot even be detected, resulting in a large number of detection blind areas, resulting in low inspection efficiency.

\subsection{The degree of intelligence is not enough}

Due to the complex working environment, the existing transmission line inspection robot cannot accurately identify all kinds of devices due to the variety of devices to maintain the stability and insulation of the line, so it cannot take the corresponding line crossing device, resulting in the line inspection task cannot be completed smoothly. However, for the substation inspection robot, it cannot respond to the environmental changes timely and accurately in the process of inspection, and cannot normally move in case of placing safety fence and personnel walking. These conditions show that the existing inspection robots are not adaptable to the complex scene environment and have insufficient ability to deal with emergencies independently.

To sum up, the research of higher intelligent power inspection robot is the need of the development of power system on the one hand, and the promotion of the development of new technology on the other hand. It can be said that the power inspection robot technology is ushering in the spring of rapid development.

\section{GIS MONITORING SYSTEM BASED ON INSPECTION ROBOT}

\subsection{Structure of GIS orbital robot}

In this paper, a GIS monitoring system based on rail robot is proposed, as shown in Figure 1, including: upper computer, determine the inspection track of the rail robot to detect GIS, generate detection tasks and transmit them to the rail robot; determine the detection positions of multiple GIS, and install multiple RFID tags and multiple UHF partial discharge sensors in GIS according to the detection positions of multiple GIS determined by the upper computer The vibration signals of high frequency, partial discharge and partial discharge are transmitted by ultrasonic and GIS signals.

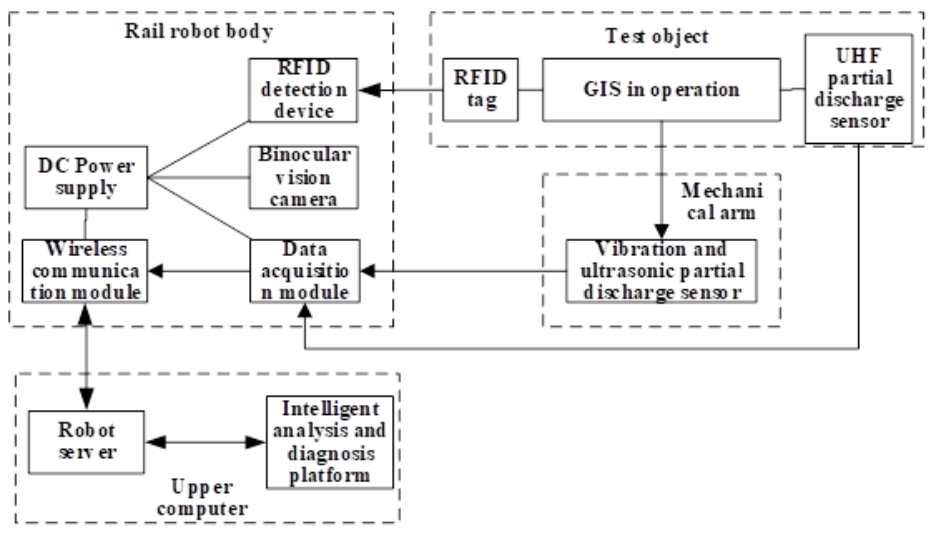

Fig. 1. Structure diagram of GIS monitoring system in operation based on rail robot

The rail robot receives the detection task of the upper computer, obtains the inspection track, and inspects the GIS according to the inspection track. Through the RFID detection device of the rail robot, the RFID tag installed on the GIS is identified, and the detection position of the GIS is determined. Through the binocular vision camera of the rail robot, the detection position is accurately positioned, and the precise positioning point of GIS is determined, and the rail robot is started The vibration sensor and ultrasonic partial discharge sensor installed on the manipulator of GIS are close to the precise positioning point of GIS, and pre-set pressing force is applied to the precise positioning point of GIS. Vibration sensor and ultrasonic partial discharge sensor are used to collect vibration signal and ultrasonic partial discharge signal of GIS, and UHF partial discharge signal of UHF partial discharge sensor are collected The signal is transmitted to the upper computer.

\subsection{Acquisition of vibration signal of GIS}

Vibration signal acquisition includes acceleration sensor, charge amplifier, data acquisition card, etc. It includes vibration pick-up link and acquisition system. The 
measured mechanical vibration is converted into electrical signal through coupling with vibration sensor on GIS shell. The data acquisition is completed by a data acquisition card including voltage amplifier, charge amplifier, integral circuit, differential circuit and filter circuit.

GIS vibration signal has many useful information to represent the running state of equipment. When GIS is in normal operation, the vibration signal component is single, which is mainly twice the fundamental frequency signal. When the mechanical fault occurs in GIS, the vibration signal of GIS equipment is collected by vibration sensor for analysis and processing, and different frequency components of different components are separated, which can effectively realize fault analysis

\subsection{UHF and ultrasonic partial discharge signal acquisition}

The basic scheme of collecting and analyzing UHF and ultrasonic PD signals is to use UHF Sensors to detect PD in GIS, and determine the approximate range of discharge power (1-2 gas chambers). Then, UHF sensor and ultrasonic sensor are used for secondary positioning analysis to achieve accurate positioning of insulation defects. The fusion of two PD signal acquisition methods can effectively eliminate the field interference and improve the accuracy of identification.

According to the structural characteristics of GIS equipment, the bisector positioning method is preliminarily considered for ultrasonic positioning analysis. The specific steps are as follows:

a) Two UHF external sensors are used to roughly locate the partial discharge (PD) and determine the approximate range of $\mathrm{PD}$ power supply;

b) One UHF external sensor is fixed on the insulator near the discharge source determined after the first positioning, and then two ultrasonic sensors are used to detect both sides of the insulator. By comparing and analyzing the detection signal amplitude, the gas chamber where the partial discharge source is located can be determined;

c) The ultrasonic sensor is moved along the longitudinal direction on the outer wall of the gas chamber where the partial discharge is located. The ultrasonic signal time delay is calculated by taking the detection signal time of UHF external sensor as the time base point. When the time delay of two detection points is the same, that is, the partial discharge source is located on the vertical bisector (GIS radial plane) between the two points;

d) The ultrasonic sensor is moved along the outer wall of GIS on the determined radial plane of GIS. When the time delay of the two detection points is the same, that is, the partial discharge source is located on the vertical bisector line between the two points;

e) When the sensor is placed at the intersection of the vertical bisector and the outer wall, the accurate position of the PD source can be calculated according to the time delay and ultrasonic propagation velocity.

\subsection{GIS intelligent diagnosis based on deep learning}

The partial discharge signal and vibration signal collected by GIS equipment are only the signal description, and the digital information for computer is formed by sampling and collecting. Then, according to the existing prior knowledge, the deep learning algorithm is used to map the collected equipment status signal with the equipment status, and the knowledge is stored in the computer. Finally, reasoning and analysing the formed knowledge to complete the final intelligent diagnosis of GIS equipment status.

\subsection{Workflow of GIS orbital robot}

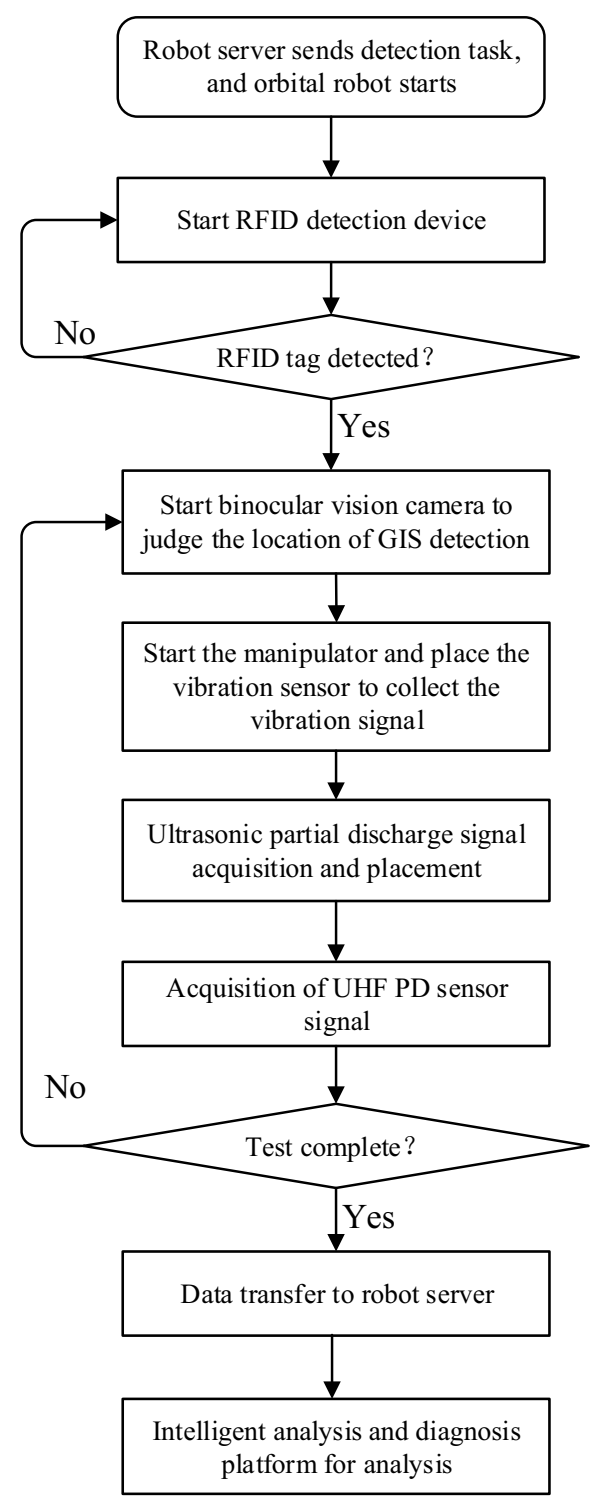

Fig. 2. Work flow chart of GIS monitoring system in transit based on rail robot

The robot server determines and issues the detection.

In the detection process, the rail robot starts the RFID detection device to judge whether the preset RFID tag on the GIS is detected. If the RFID tag is not detected, the robot will continue to move. If the tag is detected, the detection program will start. 
Firstly, the binocular vision camera is started to judge the position of GIS live detection point, and then the mechanical arm is started. The camera assists the mechanical arm to attach the vibration sensor to the GIS vibration detection point and apply a certain pressure to collect the vibration signal. Then, the mechanical arm sticks the ultrasonic partial discharge sensor to the GIS partial discharge detection point and collects the ultrasonic partial discharge signal, and then collects the UHF office through wireless way Put the signal from the sensor.

After the acquisition of vibration, ultrasonic partial discharge and UHF partial discharge signals, the robot continues to move to the next detection point for detection until the live detection of all detection points is completed. After all the detection is completed, the robot body transmits the detection data to the robot server of the upper computer through the wireless communication module, and the intelligent analysis and diagnosis platform running on it analyzes the detection data to determine whether there are defects or faults.

\section{Conclusion}

The monitoring system provided in this paper is highly integrated. By combining RFID tag, binocular vision positioning and mechanical arm, the rail robot can realize the full coverage of live detection of running GIS, and effectively improve the detection efficiency of running GIS.

The application of portable robot and intelligent live detection module in substation can greatly reduce the workload of inspection personnel, improve the frequency and quality of inspection, enhance the safety of power grid, equipment and personnel, and realize the continuous management of operation data trace, and ultimately improve the operation and maintenance management of the whole power grid Management ability. The application of GIS Substation main structure modularization and robot track and substation bracket integrated structure design can improve the integration of substation and facilitate centralized management

We thank the State Grid Corporation of Science and Technology Project Research And Application of GIS
Equipment Detection Technology Based on Portable Rail Robot (grant number 5200-201919057A-0-0-00).

\section{References}

1. Liu Q, Qin X, Yin S, et al. Structural Parameters Optimal Design AndAccuracy Analysis for Binocular Vision Measure System[C]// IEEE/ ASMEInternational Conference on Advanced Intelligent Mechatronics, Xi'an: IEEE, 2008:156161.

2. DREISBUSCH K, KRANZ H G. SCHNETTLER A. Determination of failure probability prognosis based on PD-diagnostics in GIS [J]. IEEE Transactions on Dielectrics and Electrical Insulation 2014 15(6): 1704-1707.

3. Weidong Liu,Shaohai Zhang,Shaosheng Fan. A Visual Navigation Method of Substation Inspection Robot[A]. IEEE Beijing Section, China.Proceedings of the 2016 IEEE International Conference on Progress in Informatics and Computing(PIC) [C]. IEEE Beijing Section, China, 2016: 6.

4. Li Shengfang, Hou Xingzhe. Research on the AGV Based Robot System Used in Substation Inspection[P]. Power System Technology, 2006. PowerCon 2006. International Conference on, 2006.

5. Peng Wei, Zhiqiang Zhang, Chunxi Zhang, Bingqiang Li, Qiang Du. Design of Navigation System for the Inspection Robot in Unattended Operation Substation[P]. Power and Energy Engineering Conference (APPEEC), 2010 AsiaPacific,2010.

6. ZHAO Xiaoyu, XU Zhengfei, FU Yuan. A foreign object detection algorithm suitable for inspection robot in smart substation[J]. Modern electronics technique, 2015, 38(10): 124-127.

7. LI Yuming, LIU Yongcheng, ZHANG Wenmin, et al. Design and development of intelligent inspection robot in valve hall [J]. Information technology \& informatization , 2014(10): 184-187. 